\title{
PIK3CA mutations define favorable prognostic biomarkers in operable breast cancer: a systematic review and meta-analysis
}

This article was published in the following Dove Press journal:

OncoTargets and Therapy

10 April 2014

Number of times this article has been viewed

\section{Yi-Rong Liu* \\ Yi-Zhou Jiang* \\ Wen-Jia Zuo \\ $\mathrm{Ke}-\mathrm{Da} \mathrm{Yu}$ \\ Zhi-Ming Shao}

Department of Breast Surgery, Cancer Center and Cancer Institute, Shanghai Medical College, Fudan University, Shanghai, People's Republic of China

*These authors contributed equally to this publication
Correspondence: Zhi-Ming Shao Department of Breast Surgery, Cancer Center and Cancer Institute, Fudan University, 399 Ling-Ling Road, Shanghai, 200032, People's Republic of China

Fax +862I 64434556

Email zhimingshao@yahoo.com
Background: Mutations of the $\mathrm{p} 110 \alpha$ catalytic subunit of phosphatidylinositol 3-kinase (PIK3CA) are among the most common genetic aberrations in human breast cancer. At present, controversy exists concerning the prognostic value of the mutations.

Methods: We performed a systematic review and meta-analysis to clarify the association between PIK3CA mutations and survival outcomes. A comprehensive, computerized literature search of PubMed, Web of Science databases, the Chinese Biomedical Literature Database, and Wangfang Data until August 27, 2013 was carried out. Eligible studies were included according to specific inclusion criteria. Pooled hazard ratio was estimated by using the fixed effects model or random effects model according to heterogeneity between studies.

Results: Eight eligible studies were included in the analysis, all of which were retrospective cohort studies. The overall meta-analysis demonstrated that the PIK3CA mutations were associated with better clinical outcomes (hazard ratio $0.72 ; 95 \%$ confidence interval: $0.57-0.91$; $P=0.006$ ). None of the single studies materially altered the original results and no evidence of publication bias was found. Further subgroup analysis of mutations in exons 9 and 20 did not show statistical significance.

Conclusion: PIK3CA mutations in operable primary breast cancer indicate a good prognosis. Further studies should be conducted to investigate the effect of PIK3CA mutations on clinical outcomes in different histologic types, different molecular subtypes of breast cancer, and different exons of PIK3CA.

Keywords: early breast cancer, p110g catalytic subunit of phosphatidylinositol 3-kinase, somatic mutations, prognosis

\section{Introduction}

Breast cancer is the most common malignancy among females in both developing and developed countries, with over 1.3 million cases diagnosed annually., ${ }^{1,2}$ With a more comprehensive understanding of the cancer and new protocols for treatment, the outcome of breast cancer patients has improved in the past few decades. However, breast cancer still remains the fifth leading cause of overall cancer deaths and the most frequent cause of cancer deaths in women. ${ }^{1,2}$ Identifying potential biomarkers that could serve as prognostic factors for breast cancer patients is crucial for individual treatment. Several biomarkers have been demonstrated to affect the survival of breast cancer patients so far, including P27, ${ }^{3}$ vascular endothelial growth factor (VEGF), ${ }^{4}$ cyclooxygenase-2 (COX-2), ${ }^{5}$ B cell lymphoma 2 protein (BCL-2), ${ }^{6}$ and cyclin D1. ${ }^{7}$

The phosphatidylinositol 3-kinase (PI3K)/AKT signaling pathway plays a key role in cell growth, protein translation, autophagy, metabolism, and cell survival; ${ }^{8-10}$ 
thus, tight regulation of the pathway is paramount to ensure that cellular inputs are integrated for appropriate cellular outcomes. To date, alternations in the components of the signaling pathway, including activating mutations of the $\mathrm{p} 110 \alpha$ catalytic subunit of PI3K (PI3KCA) and phosphatase and tensin homolog loss, have been identified in a wide spectrum of human cancers. ${ }^{11,12}$ The mutations of PIK3CA have been reported in $8 \%-40 \%$ of breast cancer patients, ${ }^{13-22}$ while the vast majority, comprising approximately $90 \%$ of cases, are clustered at two hot-spot regions in exon 9 and exon 20, which encode the helical and kinase domains, respectively. ${ }^{14,21-23}$ Obviously, PIK3CA mutations are among the most common genetic aberrations in human breast cancer, and their relationship with clinicopathologic characteristics has been reported. Generally, the mutations are more frequent in estrogen receptor (ER)-positive and human epidermal growth factor receptor-2 (HER2)-negative patients, ${ }^{13-16,18,20,24-26}$ but the data are not consistent. Controversy also exists concerning the prognostic value of $P I K 3 C A$ mutations. Some researchers reported that the mutations correlated with favorable outcome, ${ }^{14-17}$ while others reported the opposite results. ${ }^{27-30}$ Moreover, some authors discovered a difference in prognostic values of mutations in exon 9 and exon $20 .^{13,28,30}$

With the aim to clarify the association between PIK3CA mutations and prognosis of breast cancer patients, we conducted the first comprehensive meta-analysis of published literature on this topic in patients with operable primary breast cancer and evaluated the prognostic value of $P I K 3 C A$ mutations on disease-free survival (DFS) and overall survival (OS). To gain better insight into the underlying mechanism of the relationship, we conducted subgroup analyses of mutations in exon 9 and exon 20 of PIK3CA.

\section{Materials and methods Literature search}

A comprehensive, computerized literature search of PubMed, Web of Science databases, the Chinese Biomedical Literature Database, and Wangfang Data until August 27, 2013 was carried out. Potentially relevant studies were identified using breast cancer (ie, "breast cancer", "breast carcinoma", "breast neoplasm") and PIK3CA (ie, "phosphoinositide-3kinase catalytic, alpha polypeptide", "PI 3-kinases catalytic, alpha polypeptide", "PI3KCA") groups of search terms. The reference lists from relevant papers, especially from review articles, were checked to identify studies overlooked in the original search. This systematic review and meta-analysis was planned, conducted, and reported in adherence to the standards of quality for reporting meta-analyses. ${ }^{31}$ A flow- chart of the literature search, study selection, and results of each step is presented in Figure 1.

\section{Eligibility criteria}

All the citations from the initial search were subsequently screened for eligibility. Studies meeting all of the following inclusion criteria were deemed eligible and included in the analysis: 1) study was published in English; 2) study explored the relation between $P I K 3 C A$ mutations and clinical outcome of operable primary breast cancer; 3 ) the outcomes to assess prognosis could be presented as one or more of the following: OS, DFS, metastasis-free survival (MFS), relapse-free survival, time to distant metastasis, and breast cancer-specific survival (BCSS); and 4) hazard ratio (HR) with 95\% confidence intervals (CIs) (or sufficient data by which these could

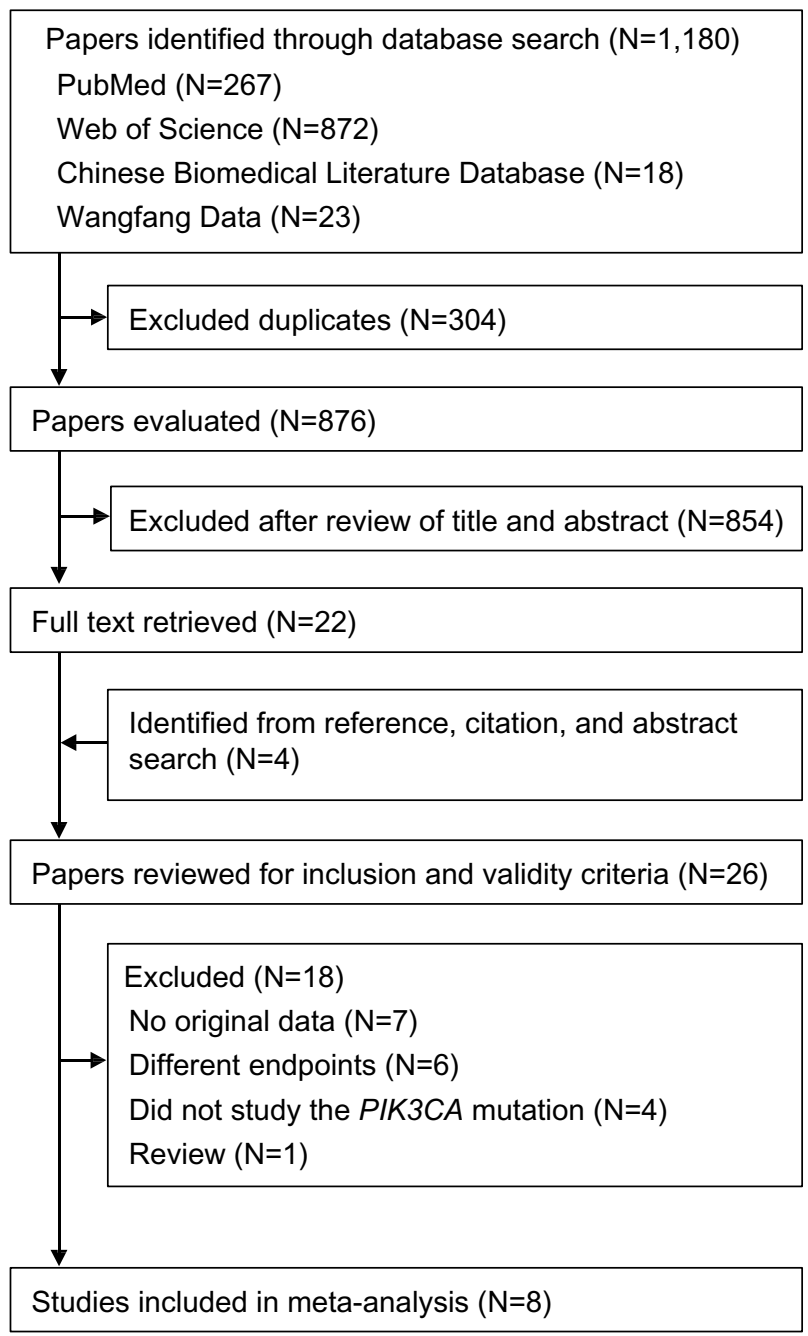

Figure I The literature search process.

Notes: One thousand one hundred and eighty studies were identified in the primary literature search. Twenty-six potentially relevant studies were further evaluated and eight studies were finally included in the analysis according to the inclusion criteria. 
be calculated) was reported. All studies not satisfying the inclusion criteria and the data obtained from review, animal experiments, or cell line studies were excluded.

Study quality was assessed using the Newcastle-Ottawa Scale. $^{32}$

\section{Data extraction and outcomes}

Two reviewers (YZJ and YRL) independently assessed studies for inclusion, and disagreements were resolved by discussion between the two. If these two reviewers could not reach a consensus, a third reviewer (KDY) was consulted to resolve the dispute and a final decision was made by the majority of votes. The following information about each eligible study was recorded: first author names; journal and year of publication; patient nationality; total number of patients studied for PIK3CA mutations; median age of patients (with range, if possible) at diagnosis; the stage or type of breast cancer; treatment protocols (besides mastectomy, lumpectomy, or any other kind of breast cancer surgery); median follow-up time (with range, if possible); number of PIK3CA-mutant patients; number of mutations in exon 9 or 20; the percentage of ER+ and HER2+ patients with PIK3CA mutations; and the clinical outcomes with HR and 95\% CI. MFS, PFS, breast cancer-specific survival, or time to distant metastasis in most studies had the same or similar definition to DFS, thus were treated as DFS when appropriate. When both the univariate and multivariate analyses were conducted, only the result of the latter is included in the present study.

\section{Statistical analysis}

Heterogeneity between studies was evaluated by $Q$-test and $I^{2}$, and either a $P$-value $<0.10$ or $I^{2}>50 \%$ was considered statistically significant. The pooled HR was estimated using the fixed effects model unless heterogeneity was found and was unexplainable, in which situation, the random effects model was applied. As different prognostic values of exon 9 and exon 20 mutations were reported, HR was also evaluated for each type of mutation. Sensitivity analysis was performed by omitting one study at a time to check if the inclusion criteria affected the final results. Potential bias of publication was examined by using the Begg's funnel plot and Egger's linear regression test $(P<0.05$ considered to be statistically significant). All statistical analysis performed in this study was carried out using Stata software (V 12.0; StataCorp LP, College Station, TX, USA).

\section{Results}

The process of the literature search and the result of each step are presented in Figure 1. One thousand one hundred and eighty studies were identified in the primary literature search, of which 26 potentially relevant studies were further evaluated after review of their titles and abstracts. A total of eight studies were included in this study according to the inclusion criteria. ${ }^{13-20}$ The main characteristics of the eligible studies, all of which were retrospective cohort studies, are shown in Table 1. The analyzed studies were published between 2007 and 2013. Six studies reported the results of outcome analysis of pooled mutations in exon 9 or exon 20 with HR and 95\% CI (or sufficient data by which these could be calculated), ${ }^{14-19}$ while four studies analyzed mutations in exon 9, and three studies reported mutations in exon 20. One study contained three subsets with a total of 1,352 patients for PIK3CA mutations, but only 342 patients with untreated lymph node-negative breast cancer were studied for the relationship between $P I K 3 C A$ mutations and MFS. ${ }^{20}$

The mutation rates of $P I K 3 C A$ in breast cancer patients from retrieved studies ranged from $25.7 \%-33.4 \%$. Of the eight studies analyzed, six presented more mutations in exon 20 than in exon $9(45.8 \%-65.6 \%$ versus $28.1 \%-42.8 \%)$, but two reported the opposite results $(30.4 \%-46.7 \%$ versus $53.3 \%-69.6 \%$ ). Data associated with the percentage of ER+ and HER2+ patients with PIK3CA mutations were also recorded. Six of the eligible studies showed that PIK3CA-mutant tumors were more likely to be $\mathrm{ER}+$ and HER2-, while the remaining two eligible studies focused on a specific subset of tumors (one on ER+ HER2-, the other on HER2+).

Figure 2A presents the forest plot of meta-analysis for OS and DFS, including HRs, 95\% CIs, and the weight of each study in the analysis. As the heterogeneity between studies was not statistically significant $\left(I^{2}=0.0 \% ; P=0.538\right)$, the fixed effects model was applied. The combined HR was 0.72 (95\% CI: $0.57-0.91 ; P=0.006)$. To further test the robustness of our study, we performed sensitivity analysis by omitting one study each time. We found that no single study altered the original results significantly (Figure 2B). Funnel plot and Egger's test showed no evidence of publication bias $(P=0.466)$.

Several studies demonstrated the inconsistent prognostic value of $P I K 3 C A$ mutations in exon 9 and exon 20, thus we performed further meta-analysis in the subgroup of patients with mutations in either exon. Two papers included in the previously pooled mutations analysis also contained data for outcome analysis in exon 9 and exon 20. One paper reporting the results for exon 9 and exon 20, respectively, but without pooled results, was also included. Another eligible 
Table I Characteristics of eligible studies

\begin{tabular}{|c|c|c|c|c|c|c|c|c|}
\hline Study, year & Country & $\begin{array}{l}\text { Number } \\
\text { of patients }\end{array}$ & $\begin{array}{l}\text { Median } \\
\text { patient age, } \\
\text { years }\end{array}$ & Stage & Treatment & $\begin{array}{l}\text { Median } \\
\text { follow-up } \\
\text { time, months }\end{array}$ & $\begin{array}{l}\text { Sample } \\
\text { type }\end{array}$ & $\begin{array}{l}\text { Mutation } \\
\text { detection } \\
\text { method }\end{array}$ \\
\hline $\begin{array}{l}\text { Barbareschi } \\
\text { et al, }{ }^{13} 2007\end{array}$ & Italy & 161 & $\begin{array}{l}62 \\
\text { (range 17-89) }\end{array}$ & PBC & Chem or horm & $N R$ & FTS & SSCP \\
\hline $\begin{array}{l}\text { Maruyama } \\
\text { et al, }{ }^{14} 2007\end{array}$ & Japan & 188 & NR & $\mathrm{PBC}$ & $\begin{array}{l}\text { Chem or horm } \\
\text { or chem + horm }\end{array}$ & $\begin{array}{l}64 \\
\text { (range 38-88) }\end{array}$ & FTS & $A B I$ \\
\hline $\begin{array}{l}\text { Kalinsky } \\
\text { et al, }{ }^{15} 2009\end{array}$ & USA & 590 & $\begin{array}{l}58 \\
\text { (range 27-89) }\end{array}$ & $\mathrm{PBC}$ & NR & 153 & FFPE & $\begin{array}{l}\text { ARRAY } \\
\text { and SS }\end{array}$ \\
\hline $\begin{array}{l}\text { Bozhanov } \\
\text { et al, }{ }^{16} 2010\end{array}$ & Bulgaria & 145 & $\begin{array}{l}\text { NR } \\
\text { (range 29-88) }\end{array}$ & $\mathrm{PBC}$ & NR & $\begin{array}{l}69 \\
\text { (range II-96) }\end{array}$ & FTS & DS \\
\hline Loi et al, ${ }^{17} 2010$ & USA & 173 & NR & I-III & Horm & 115 & FTS & SSCP \\
\hline $\begin{array}{l}\text { Cizkova } \\
\text { et al, }{ }^{18} 2012\end{array}$ & France & 452 & $\begin{array}{l}61.6^{\mathrm{b}} \\
\text { (range 3I-9I) }\end{array}$ & $\mathrm{PBC}$ & $\begin{array}{l}\text { Chem or horm } \\
\text { or chem + horm }\end{array}$ & 120 & FTS & $A B I$ \\
\hline $\begin{array}{l}\text { Jensen } \\
\text { et al, }{ }^{19} 2012\end{array}$ & Denmark & 237 & NR & I-III & $\begin{array}{l}\text { Chem }+\mathrm{T} \text { or } \\
\text { chem }+ \text { horm }+\mathrm{T}\end{array}$ & 67 & FFPE & DiS and PS \\
\hline $\begin{array}{l}\text { Ramirez-Ardila } \\
\text { et al, }{ }^{20} 2013\end{array}$ & $\begin{array}{l}\text { The Netherlands } \\
\text { and Belgium }\end{array}$ & $1,352^{\mathrm{a}}$ & NR & $\begin{array}{l}\mathrm{PBC} \\
\text { or } A B C\end{array}$ & $\begin{array}{l}\text { Untreated } \\
\text { or endo }\end{array}$ & NR & $\begin{array}{l}\text { FTS or } \\
\text { FFPE }\end{array}$ & Sna \\
\hline
\end{tabular}

Notes: aThe study contained three subsets. Only 342 patients with untreated lymph node-negative breast cancer were studied for the relationship between PIK3CA mutation and metastasis-free survival; ' ${ }^{b}$ mean age of the study cases; 'the percentage of ER+ patients in all PIK3CA-mutant patients; 'the percentage of HER2+ patients in all PIK3CAmutant patients; 'evaluated by the Newcastle-Ottawa Scale. ${ }^{33}$ Sequenom MassARRAY ${ }^{\circledR}$ : University of Arizona Genetics Core, Tucson, AZ, USA; SNaPshot ${ }^{\circledR}$ Multiplex System: Life Technologies, Carlsbad, CA, USA; ABI automated sequence: Applied Biosystems, Inc., CA, USA.

Abbreviations: $A B C$, advanced breast cancer; $A B I, A B I$ automated sequencer; ARRAY, Sequenom MassARRAY system; chem, chemotherapy; DFS, disease-free survival; DiS, dideoxynucleotide sequencing; DS, direct sequencing; endo, endocrine therapy; ER, estrogen receptor; FFPE, formalin-fixed, paraffin-embedded tissue sample; FTS, frozen tissue sample; HER2, human epidermal growth factor receptor-2; horm, hormonotherapy; NR, not reported; OS, overall survival; PBC, primary breast cancer; PIK3CA, pI I0 $\alpha$ catalytic subunit of phosphatidylinositol 3-kinase; PS, pyrosequencing; Sna, SNaPshot Multiplex System sequencing; SS, Sanger sequencing; SSCP, single-strand conformation polymorphism; T, trastuzumab.

paper with data available only for exon 9 was added to the analysis of that subgroup. Therefore, four and three papers were included for analysis of mutations in exon 9 and exon 20 , respectively.

Figure 3A shows the forest plot of meta-analysis of mutations in exon 9 . Heterogeneity was statistically significant among the four studies $\left(I^{2}=68.8 \% ; P=0.022\right)$. The random effects model was performed, and the combined HR was 1.36 (95\% CI: $0.65-2.83 ; P=0.253$ ). To explore the origin of the heterogeneity, we performed sensitivity analysis. This showed that the study by Barbareschi et al substantially impacted the pooled HR (Figure 3B). After omitting the study, heterogeneity was no longer observed $\left(I^{2}=0.0 \% ; P=0.688\right)$ and the revised HR was 0.95 (95\% CI: $0.62-1.47 ; P=0.831) .{ }^{13}$

The analysis was repeated for mutations in exon 20 (Figure 4). The fixed effects model was performed, with a combined HR of 0.98 (95\% CI: 0.68-1.42; $P=0.931$ ). There was no evidence of heterogeneity among studies $\left(I^{2}=0.0 \% ; P=0.899\right)$.

\section{Discussion}

We performed a systematic review and meta-analysis to clarify the relationship between PIK3CA mutations and survival outcome. Eight eligible studies with PIK3CA mutation rates ranging from $25.7 \%-33.4 \%$ were included in the analysis. Our study demonstrates that PIK3CA mutations are associated with improved clinical outcomes in operable primary breast cancer, but the associations are not statistically significant in the subgroup analysis of mutations in exon 9 or exon 20 of PIK3CA.

PI3K is among the most important downstream molecules in the pathway of tyrosine kinase growth factor receptors, and one of the most promising targets for translational research in oncology. ${ }^{33}$ Once activated, PI3K can catalyze the phosphorylation of inositol lipids to produce phosphatidylinositol-3,4,5-trisphosphate. ${ }^{34}$ Phosphatidylinositol3,4,5-trisphosphate activates the serine/threonine kinase AKT, which, in turn, regulates several signaling pathways that control cell survival, apoptosis, proliferation, motility, growth, cytoskeletal rearrangement, migration, and cancer metastasis. ${ }^{35-38}$ Thus the activation of the PI3K/AKT pathway may have pathogenic effects in human cancer, and some of the derangements in the pathway could be candidate targets for therapy. ${ }^{8,11,33,35-38}$

The typical form of PI3K is as a heterodimer with an $85 \mathrm{kDa}$ regulatory subunit and a $110 \mathrm{kDa}$ catalytic subunit. The $85 \mathrm{kDa}$ regulatory subunit can bind to phosphotyrosine residues of activated growth factors, inducing activation of 


\begin{tabular}{|c|c|c|c|c|c|c|c|}
\hline $\begin{array}{l}\text { Mutation } \\
\text { type }\end{array}$ & $\begin{array}{l}\text { Number of } \\
\text { PIK3CA-mutant } \\
\text { patients }(\%)\end{array}$ & $\begin{array}{l}\text { Number of } \\
\text { mutations } \\
\text { in exon } 9(\%)\end{array}$ & $\begin{array}{l}\text { Number of } \\
\text { mutations } \\
\text { in exon } 20(\%)\end{array}$ & $E R+(\%)^{c}$ & HER 2+ (\%) ${ }^{d}$ & Outcome & $\begin{array}{l}\text { Study } \\
\text { qualitye }\end{array}$ \\
\hline Missense & $45(28.0)$ & $24(53.3)$ & 21 (46.7) & 84.4 & 17.8 & DFS & 8 \\
\hline Missense & $54(29)$ & $17(3 \mid .5)$ & $29(53.7)$ & 77.8 & 18.8 & DFS & 8 \\
\hline Missense & $192(32.5)$ & $54(28.1)$ & $88(45.8)$ & 76.2 & 3.6 & DFS & 7 \\
\hline Missense & $46(31.7)$ & $18(39.1)$ & $28(60.9)$ & 53.3 & 27.8 & OS & 7 \\
\hline Missense & $46(26.6)$ & $32(69.6)$ & $14(30.4)$ & 100 & 0.0 & DFS & 6 \\
\hline Missense & I5I (33.4) & $65(42.8)$ & $87(57.2)$ & 67.8 & 25.2 & DFS & 8 \\
\hline Missense & 61 (25.7) & $21(34.4)$ & $40(65.6)$ & 52.5 & 100 & DFS & 8 \\
\hline Missense & $423(31)$ & I73 (40.8) & $25 \mathrm{I}(59.2)$ & 91.2 & 9.8 & DFS & 8 \\
\hline
\end{tabular}

the lipid kinase activity of the $110 \mathrm{kDa}$ catalytic subunit. The catalytic subunit is encoded by the PIK3CA gene, which is amplified, overexpressed, or mutated in several human malignancies, especially in breast cancer. ${ }^{11,12,22}$ Several studies have reported $P I K 3 C A$ mutation in breast cancer patients, with great inconsistency regarding the association between mutation and clinical outcome. ${ }^{14-17,27-30}$ A meta-analysis would help to clarify this issue.

In the present study, we have demonstrated that the mutations of PIK3CA are associated with better prognosis. Only studies with operable primary breast cancer were included in the analysis. Studies researching metastatic breast cancer or other breast cancers were excluded, considering the many confounding factors that may exist in these patients, which would impair the efficacy of this study. Six eligible studies included in the analysis were without heterogeneity, and the pooled HR was 0.72 (95\% CI: $0.57-0.91 ; P=0.006)$. However, the subgroup analysis of mutations in exon 9 and exon 20 did not achieve the same result with statistical significance. Heterogeneity was significant among the studies in the subset of exon 9. Sensitivity analysis showed that the study by Barbareschi et a ${ }^{13}$ seemed to be a major source of heterogeneity. After removing this study, the heterogeneity disappeared, and the combined HR declined from 1.36 to
0.95, still without statistical significance. The disparate results before and after removing this study may be due to the high proportion of lobular tumor in exon 9 PIK3CA-mutant patients $(33.3 \%$ of exon 9 PIK3CA-mutant cases were lobular tumor, while the number for the other three studies included in this subgroup analysis were less than $9 \%$ or unknown).

Results of the subgroup analysis of exon 9 and exon 20 mutations did not agree with the result from pooled $P I K 3 C A$ mutations. There are a number of possible reasons for this. First, the numbers of studies and patients included in the subgroup analysis were relatively small, and may not be enough to tell the difference between the clinical outcomes. For mutations in exon 9, four studies with 913 patients were analyzed at first. After removing one study (that of Barbareschi et al) $)^{13}$ for its heterogeneity, the number declined to three studies with 752 patients, which was the same sample size as in the analysis of mutations in exon 20, but less than half the sample size of the pooled $P I K 3 C A$ mutation analysis. The small population could be more vulnerable to confounding factors, making it more difficult to distinguish statistical difference. Second, not all of the studies analyzing the pooled mutations conducted subgroup analyses. The studies by Loi et $\mathrm{al}^{17}$ and Jensen et $\mathrm{al}^{19}$ found that the combined mutation analysis did not have statistical significance and further 
A

Study Country $\quad H R(95 \% \mathrm{Cl}) \quad$ Weight (\%)

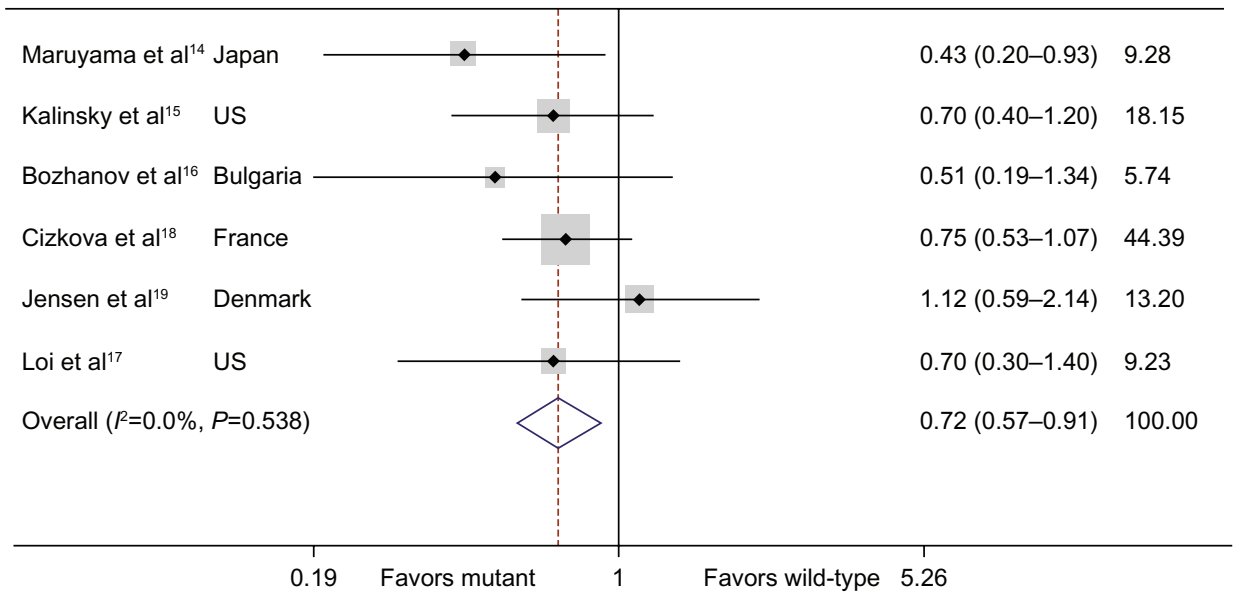

B

Meta-analysis fixed-effects estimates (exponential form)

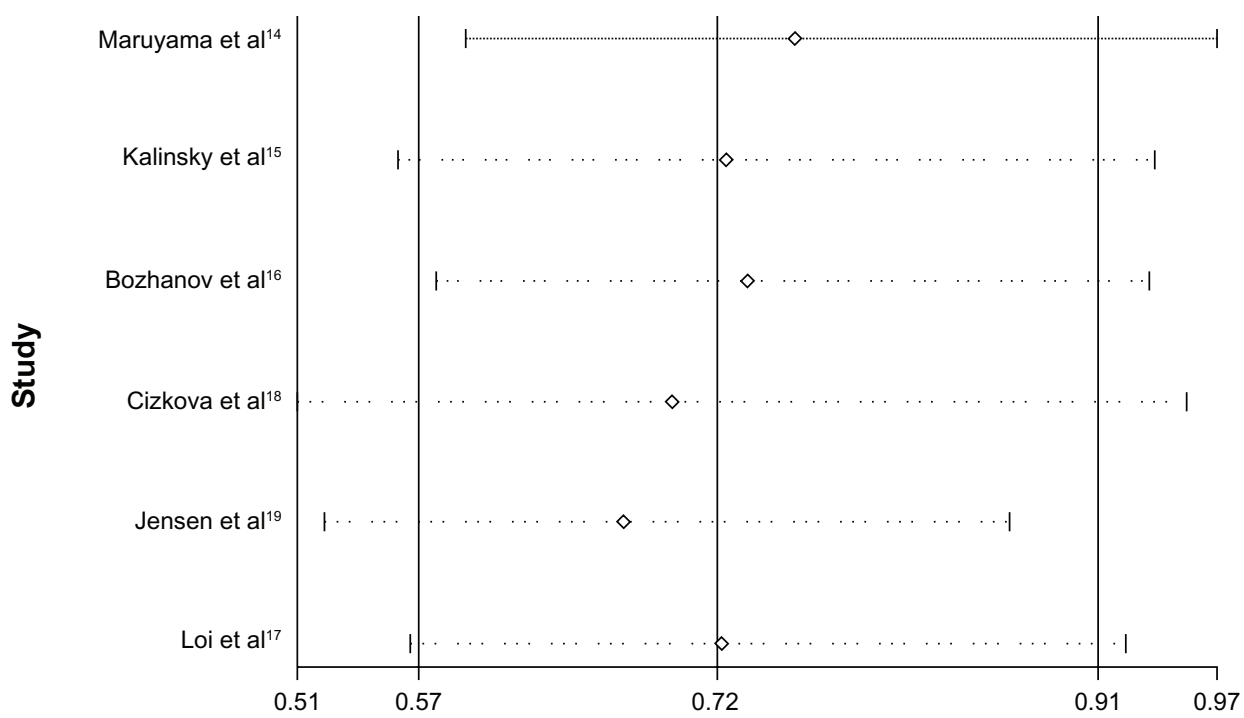

Figure 2 Individual study and overall HRs of relationships between PIK3CA mutations and treatment outcomes.

Notes: The mutations analyzed here included mutations in exon 9 and exon 20, except in the study by Maruyama et al, ${ }^{14}$ which also contained mutations in other exons. (A) Forest plots; (B) Influence of individual studies on the pooled HR.

Abbreviations: $\mathrm{Cl}$, confidence interval; HR, hazard ratio; PIK3CA, pI I0 $\alpha$ catalytic subunit of phosphatidylinositol 3-kinase.

analyzed the subgroups. In other words, studies that analyzed the subgroups tended to report statistical nonsignificance of the combined analysis. This bias could impair the final results of the subgroup meta-analysis. Furthermore, of the three studies included in the subgroup analysis, the study by Loi et $\mathrm{al}^{17}$ focused on the primary ER+/HER2- breast cancers, while Jensen et $\mathrm{al}^{19}$ studied HER2+ patients. The effect of PIK3CA mutation in different subtypes of breast cancer may vary. Future studies should scrutinize the association of PIK3CA mutations with specific molecular subtypes of breast cancer, such as luminal A, luminal B, basal-like, and HER2+ subtypes.

The following limitations of this study should be declared. First, there may be other prognostic factors not controlled in the meta-analysis. The different exons in which the mutations resided, different histologic types of cancer, hormonal receptor status, and HER2 status were not always adjusted in the eligible studies. The effect of the PIK3CA mutations on clinical outcomes may vary in patients with different clinicopathological features. However, due to 
A

Study

Country

HR $(95 \% \mathrm{Cl}) \quad$ Weight $(\%)$

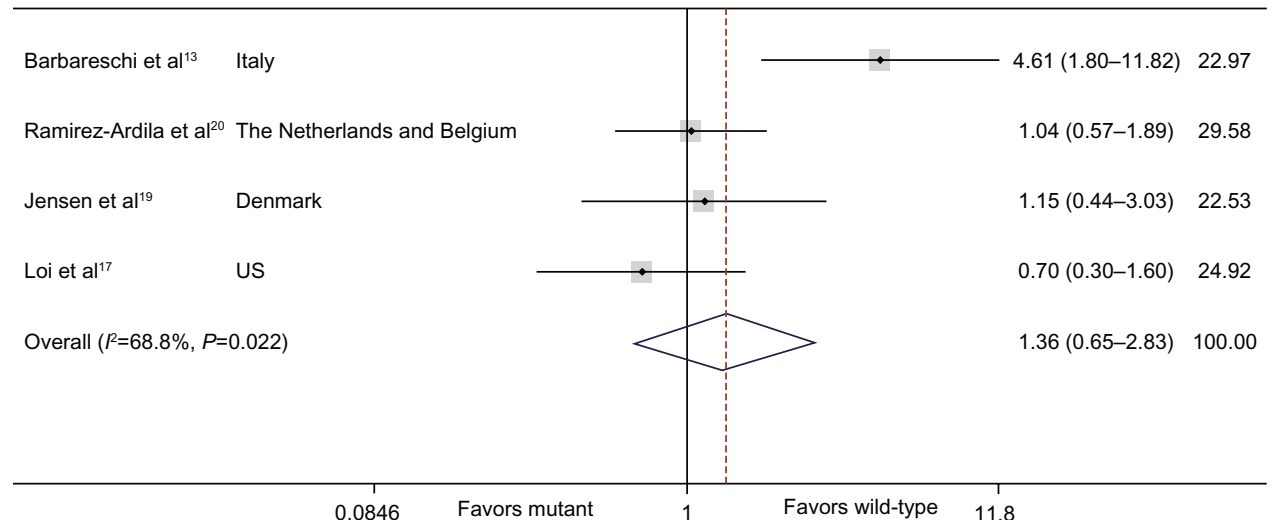

B

Meta-analysis random-effects estimates (exponential form)

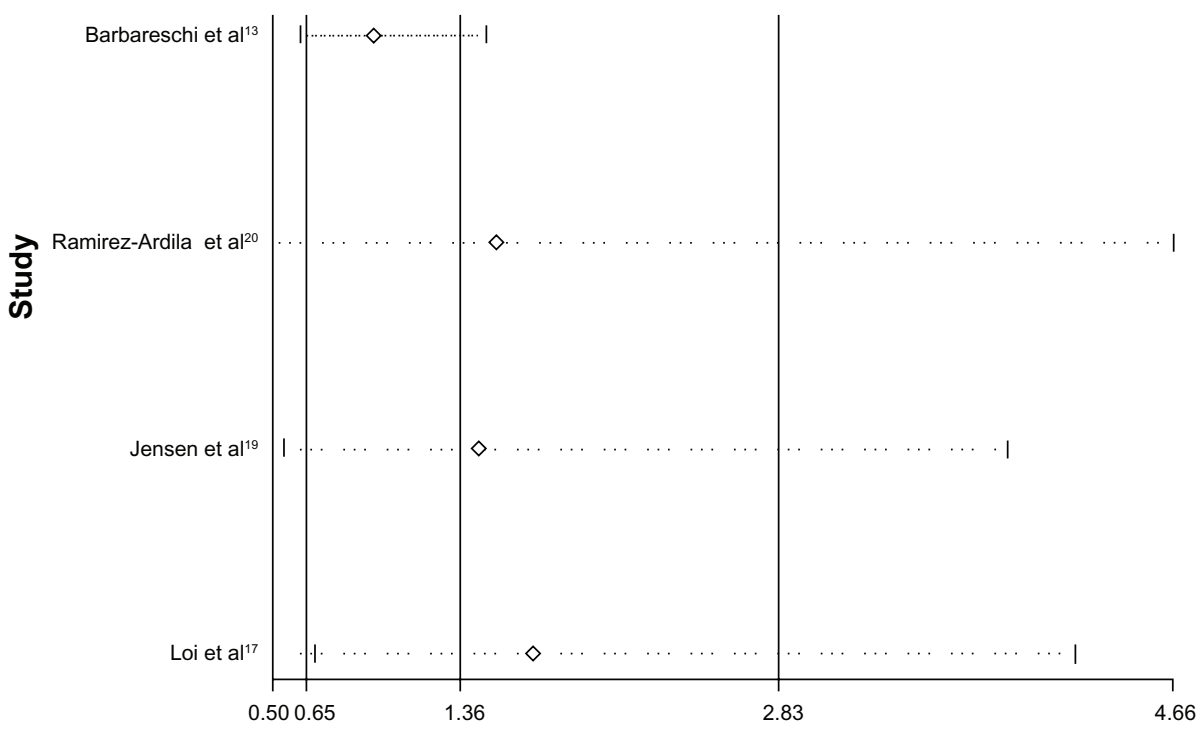

Figure 3 Individual study and overall HRs of relationships between PIK3CA mutations in exon 9 and treatment outcomes.

Notes: (A) Forest plots. Weights are from random effects analysis; (B) Influence of individual studies on the pooled HR.

Abbreviations: $\mathrm{Cl}$, confidence interval; HR, hazard ratio; PIK3CA, pI I $0 \alpha$ catalytic subunit of phosphatidylinositol 3-kinase.

the lack of sufficient patient information, we were unable to conduct sub-analyses according to different features. Second, our meta-analysis was based on data only from studies whose results have been published, and we could not obtain updated data on individual patients. Use of individual patient data could further enhance the accuracy and reduce the uncertainty of our estimates. Third, because we evaluated studies for inclusion through review of title and abstract, studies that did not mention HR and $95 \% \mathrm{CI}$ in their abstract were excluded, and this may have led to selection bias. Finally, publication bias was also a concern. It was unavoidable that some data would remain unobtainable even after we tried to identify all relevant information. However, after examining the Begg's funnel plots and performing the Egger's linear regression test, we found that the association between PIK3CA mutation and clinical outcome remained unchanged.

\section{Conclusion}

Our meta-analysis demonstrates the favorable prognostic value of PIK3CA mutations in operable primary breast cancer patients. Further studies should investigate the effects of 
A
Study
Country
$\operatorname{HR}(95 \% \mathrm{Cl})$
Weight \%

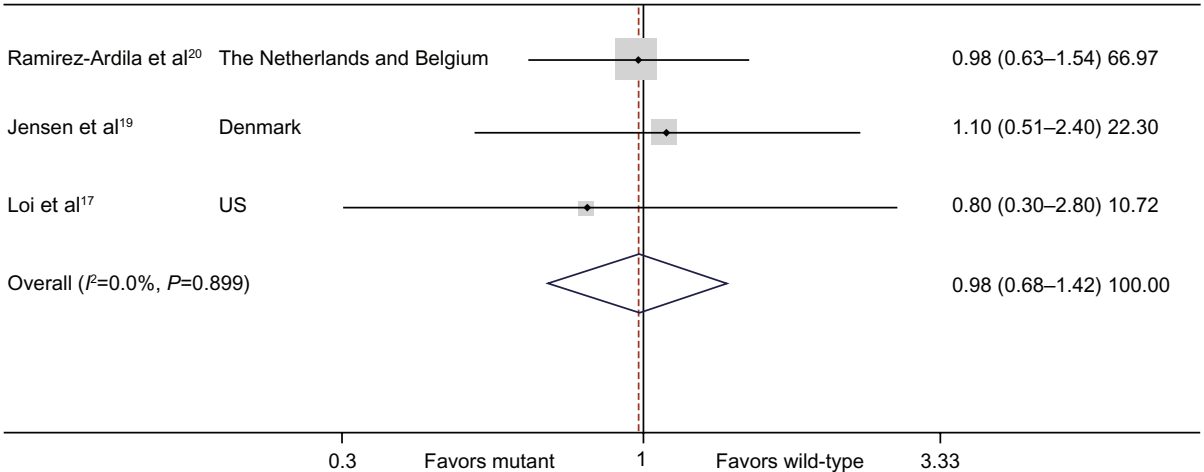

B

Meta-analysis fixed-effects estimates (exponential form)

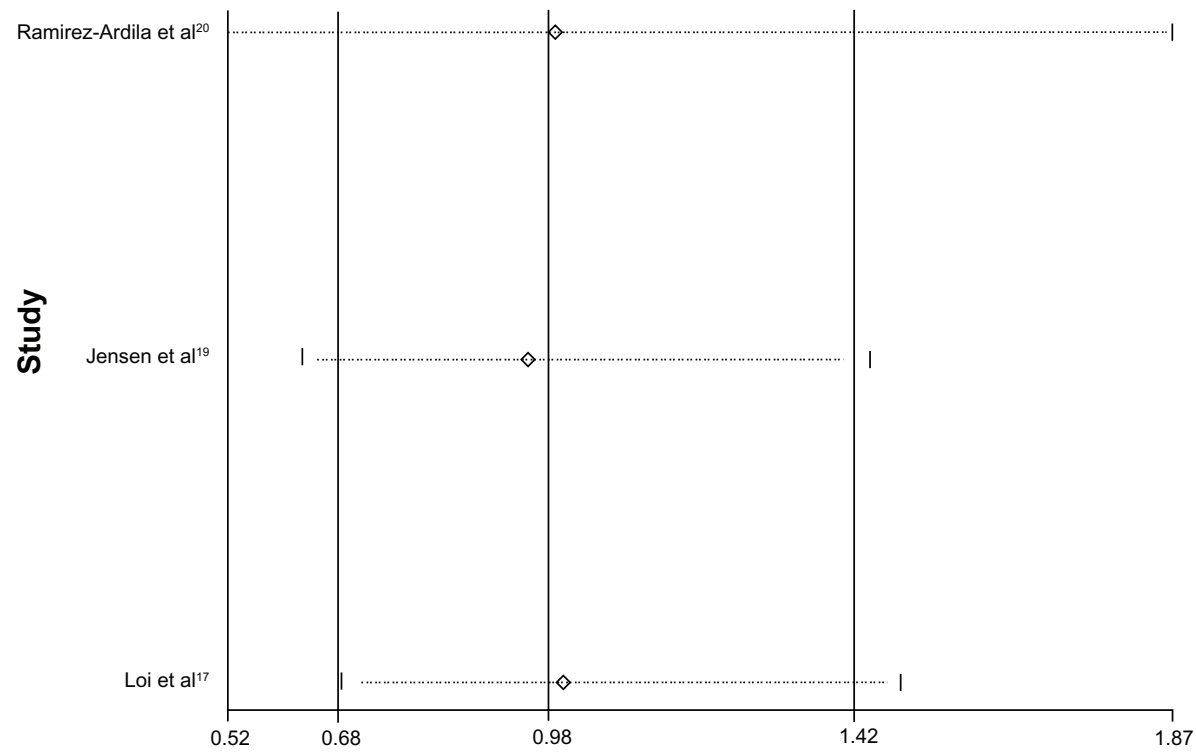

Figure 4 Individual study and overall HRs of relationships between PIK3CA mutations in exon 20 and treatment outcomes.

Notes: (A) Forest plots; (B) Influence of individual studies on the pooled HR.

Abbreviations: $\mathrm{Cl}$, confidence interval; HR, hazard ratio; PIK3CA, pIIO $\alpha$ catalytic subunit of phosphatidylinositol 3-kinase.

PIK3CA mutations on clinical outcome in different histologic types, different molecular subtypes of breast cancer, and different exons of PIK3CA.

\section{Acknowledgments}

This work was supported by grants from the National Natural Science Foundation of China (81001169, 81370075, and 81372848); the Shanghai United Developing Technology Project of Municipal Hospitals (SHDC12010116); the Shanghai Key Laboratory of Breast Cancer (12DZ2260100); and the Zhuo-Xue Project of Fudan University (for KDY). The funders had no role in the study design, data collection and analysis, decision to publish, or preparation of the manuscript. The authors are grateful to Jiong Wu, Jin-Song Lu, Guang-Yu Liu, Gen-Hong Di, and Zhen-Zhou Shen for their excellent data handling.

\section{Author contributions}

Conceived and designed the experiments: YRL, YZJ, KDY, ZMS. Performed the experiments: YRL, YZJ, KDY. Analyzed the data:YRL, YZJ, KDY, WJZ. Contributed reagents/materials/analysis tools: YRL, YZJ, KDY, WJZ. All authors took part in drafting the article or revising it critically for important intellectual content. 


\section{Disclosure}

The authors report no conflicts of interest in this work.

\section{References}

1. Cancer. Fact sheet No 297 [webpage on the Internet]. Geneva: World Health Organization; 2013 [updated February, 2014]. Available from: http://www.who.int/mediacentre/factsheets/fs297/en/. Accessed: October 30, 2013.

2. Global Cancer Facts and Figures. 2nd ed. [webpage on the Internet]. USA: American Cancer Society; 2011. Available from: http://www. cancer.org/research/cancerfactsfigures/globalcancerfactsfigures/globalfacts-figures-2nd-ed. Accessed October 30, 2013.

3. Guan X, Wang Y, Xie R, et al. p27(Kip1) as a prognostic factor in breast cancer: a systematic review and meta-analysis. J Cell Mol Med. 2010;14(4):944-953.

4. Wang J, Guo Y, Wang B, et al. Lymphatic microvessel density and vascular endothelial growth factor-C and -D as prognostic factors in breast cancer: a systematic review and meta-analysis of the literature. Mol Biol Rep. 2012;39(12):11153-11165.

5. Kim HS, Moon HG, Han W, et al. COX2 overexpression is a prognostic marker for Stage III breast cancer. Breast Cancer Res Treat. 2012; 132(1):51-59.

6. Callagy GM, Webber MJ, Pharoah PD, Caldas C. Meta-analysis confirms BCL2 is an independent prognostic marker in breast cancer. BMC Cancer. 2008;8:153.

7. Xu XL, Chen SZ, Chen W, et al. The impact of cyclin D1 overexpression on the prognosis of ER-positive breast cancers: a meta-analysis. Breast Cancer Res Treat. 2013;139(2):329-339.

8. Agarwal R, Carey M, Hennessy B, Mills GB. PI3K pathway-directed therapeutic strategies in cancer. Current Opin Investig Drugs. 2010;11(6):615-628.

9. Hennessy BT, Smith DL, Ram PT, Lu Y, Mills GB. Exploiting the PI3K/ AKT pathway for cancer drug discovery. Nature Rev Drug Discov. 2005;4(12):988-1004.

10. Meric-Bernstam F, Gonzalez-Angulo AM. Targeting the mTOR signaling network for cancer therapy. J Clin Oncol. 2009;27(13): 2278-2287.

11. Samuels Y, Waldman T. Oncogenic mutations of PIK3CA in human cancers. Curr Top Microbiol Immunol. 2010;347:21-41.

12. Chalhoub N, Baker SJ. PTEN and the PI3-kinase pathway in cancer. Annu Rev Pathol. 2009;4:127-150.

13. Barbareschi M, Buttitta F, Felicioni L, et al. Different prognostic roles of mutations in the helical and kinase domains of the PIK3CA gene in breast carcinomas. Clin Cancer Res. 2007;13(20):6064-6069.

14. Maruyama N, Miyoshi Y, Taguchi T, Tamaki Y, Monden M, Noguchi S. Clinicopathologic analysis of breast cancers with PIK3CA mutations in Japanese women. Clin Cancer Res. 2007;13(2 Pt 1):408-414.

15. Kalinsky K, Jacks LM, Heguy A, et al. PIK3CA mutation associates with improved outcome in breast cancer. Clin Cancer Res. 2009;15(16): 5049-5059.

16. Bozhanov SS, Angelova SG, Krasteva ME, et al. Alterations in p53, BRCA1, ATM, PIK3CA, and HER2 genes and their effect in modifying clinicopathological characteristics and overall survival of Bulgarian patients with breast cancer. J Cancer Res Clin Oncol. 2010;136(11): 1657-1669.

17. Loi S, Haibe-Kains B, Majjaj S, et al. PIK3CA mutations associated with gene signature of low mTORC1 signaling and better outcomes in estrogen receptor-positive breast cancer. Proc Natl Acad Sci US A. 2010;107(22):10208-10213.

18. Cizkova M, Susini A, Vacher S, et al. PIK3CA mutation impact on survival in breast cancer patients and in ER $\alpha$, PR and ERBB2-based subgroups. Breast Cancer Res. 2012;14(1):R28.
19. Jensen JD, Knoop A, Laenkholm AV, et al. PIK3CA mutations, PTEN, and pHER2 expression and impact on outcome in HER2-positive earlystage breast cancer patients treated with adjuvant chemotherapy and trastuzumab. Ann Oncol. 2012;23(8):2034-2042.

20. Ramirez-Ardila DE, Helmijr JC, Look MP, et al. Hotspot mutations in PIK3CA associate with first-line treatment outcome for aromatase inhibitors but not for tamoxifen. Breast Cancer Res Treat. 2013;139(1): 39-49.

21. Campbell IG, Russell SE, Choong DYH, et al. Mutation of the PIK3CA gene in ovarian and breast cancer. Cancer Res. 2004;64(21): 7678-7681.

22. Samuels Y, Wang Z, Bardelli A, et al. High frequency of mutations of the PIK3CA gene in human cancers. Science. 2004;304(5670):554.

23. Saal LH, Holm K, Maurer M, et al. PIK3CA mutations correlate with hormone receptors, node metastasis, and ERBB2, and are mutually exclusive with PTEN loss in human breast carcinoma. Cancer Res. 2005;65(7):2554-2559.

24. Bachman KE, Argani P, Samuels Y, et al. The PIK3CA gene is mutated with high frequency in human breast cancers. Cancer Biol Ther. 2004;3(8):772-775.

25. Boyault S, Drouet Y, Navarro C, et al. Mutational characterization of individual breast tumors: TP53 and PI3K pathway genes are frequently and distinctively mutated in different subtypes. Breast Cancer Res Treat. 2012;132(1):29-39.

26. Michelucci A, Di Cristofano C, Lami A, et al. PIK3CA in breast carcinoma: a mutational analysis of sporadic and hereditary cases. Diagn Mol Patholgy. 2009;18(4):200-205.

27. Li SY, Rong M, Grieu F, Iacopetta B. PIK3CA mutations in breast cancer are associated with poor outcome. Breast Cancer Res Treat. 2006;96(1):91-95.

28. Lai YL, Mau BL, Cheng WH, Chen HM, Chiu HH, Tzen CY. PIK3CA exon 20 mutation is independently associated with a poor prognosis in breast cancer patients. Ann Surg Oncol. 2008;15(4):1064-1069.

29. Aleskandarany MA, Rakha EA, Ahmed MA, et al. PIK3CA expression in invasive breast cancer: a biomarker of poor prognosis. Breast Cancer Res Treat. 2010;122(1):45-53.

30. Mangone FR, Bobrovnitchaia IG, Salaorni S, Manuli E, Nagai MA. PIK3CA exon 20 mutations are associated with poor prognosis in breast cancer patients. Clinics (Sao Paulo). 2012;67(11):1285-1290.

31. DerSimonian R, Laird N. Meta-analysis in clinical trials. Control Clin Trials. 1986;7(3):177-188.

32. Wells GA, Shea B, O'Connell D, et al. The Newcastle-Ottawa Scale (NOS) for assessing the quality of nonrandomised studies in meta-analyses [webpage on the Internet]. Ottawa: Ottawa Hospital Research Institute. Available from: http:/www.ohri.ca/programs/clinical_epidemiology/oxford.htm. Accessed October 30, 2013.

33. Shaw RJ, Cantley LC. Ras, PI(3)K and mTOR signalling controls tumour cell growth. Nature. 2006;441(7092):424-430.

34. Bader AG, Kang S, Zhao L, Vogt PK. Oncogenic PI3K deregulates transcription and translation. Nat Rev Cancer. 2005;5(12):921-929.

35. Yoeli-Lerner M, Toker A. Akt/PKB signaling in cancer: a function in cell motility and invasion. Cell Cycle. 2006;5(6):603-605.

36. Chin YR, Toker A. The actin-bundling protein palladin is an Akt1specific substrate that regulates breast cancer cell migration. Mol Cell. 2010;38(3):333-344.

37. Xue G, Restuccia DF, Lan Q, et al. Akt/PKB-mediated phosphorylation of Twist1 promotes tumor metastasis via mediating cross-talk between PI3K/ Akt and TGF- $\beta$ signaling axes. Cancer Discov. 2012;2(3): 248-259.

38. Xue G, Hemmings BA. PKB/Akt-dependent regulation of cell motility. J Natl Cancer Inst. 2013;105(6):393-404. 


\section{Publish your work in this journal}

OncoTargets and Therapy is an international, peer-reviewed, open access journal focusing on the pathological basis of all cancers, potential targets for therapy and treatment protocols employed to improve the management of cancer patients. The journal also focuses on the impact of management programs and new therapeutic agents and protocols on

patient perspectives such as quality of life, adherence and satisfaction. The manuscript management system is completely online and includes a very quick and fair peer-review system, which is all easy to use. Visit http://www.dovepress.com/testimonials.php to read real quotes from published authors.

Submit your manuscript here: http://www.dovepress.com/oncotargets-and-therapy-journal 